\title{
BEST EXPONENTS IN MARKOV'S INEQUALITIES
}

\author{
ALEXANDER GONCHAROV
}

Abstract. By means of weakly equilibrium Cantor-type sets, solutions of two problems related to polynomial inequalities are presented: the problem by $\mathrm{M}$. Baran et al. about a compact set $K \subset \mathbb{C}$ such that the Markov inequality is not valid on $K$ with the best Markov's exponent, and the problem by L. Frerick et al. concerning compact sets satisfying the local form of Markov's inequality with a given exponent, but not satisfying the global version of Markov's inequality with the same parameter.

Mathematics subject classification (2010): 41A17, 41A44.

Keywords and phrases: Global and local Markov's inequalities, Markov's exponents.

\section{REFERENCES}

[1] M. Altun And A. Goncharov, A local version of the Pawtucki-Pleśniak extension operator, J. Approx. Theory, 132 (2005), 34-41.

[2] M. Baran AND W. PleŚniak, Markov's exponent of compact sets in $\mathbb{C}^{n}$, Proc. Amer. Math. Soc., 123 (1995), 2785-2791.

[3] M. Baran, L. BiaŁas-Cież And B. Milówka, On the best exponent in Markov inequality, Potential Anal., 38 (2013), 635-651.

[4] L. BiaŁas And A. Volberg, Markov's property of the Cantor ternary set, Studia Math., 104 (1993), 259-268

[5] L. Bos And P. Milman, Sobolev-Gagliardo-Nirenberg and Markov type inequalities on subanalytic domains, Geom. Funct. Anal., 5 (6), (1995), 853-923

[6] L. Carles on And T. Gamelin, Complex dynamics. Universitext: Tracts in Mathematics, SpringerVerlag, New York, 1993.

[7] L. FRERICK, E. JoRdÁ AND J. WengENROTH, Tame linear extension operators for smooth Whitney functions, J. Funct. Anal., 261 (2011), 591-603.

[8] A. Goncharov, A compact set without Markov's property but with an extension operator for $C^{\infty}$ functions, Studia Math., 119 (1996), 27-35.

[9] A. Goncharov, Perfect sets of finite class without the extension property, Studia Math., 126 (1997), $161-170$.

[10] A. Goncharov, Weakly Equilibrium Cantor-type sets, to appear Potential Analysis 40 (2014), 143 161.

[11] A. Jonsson And H. WALlin, Function spaces on subsets of $\mathbb{R}^{n}$, Math. Rep. 2, 1984.

[12] A. JONSSON, Markov's inequality and zeros of orthogonal polynomials on fractal sets, J. Approx. Theory, 78 (1994), 87-97.

[13] J. LiTHner, Comparing two versions of Markov's inequality on compact sets, J. Approx. Theory, 77 (1994), 202-211.

[14] W. PAWŁUCKi AND W. PleŚNIAK, Extension of $C^{\infty}$ functions from sets with polynomial cusps, Studia Math., 88 (1988), 279-287.

[15] W. PLEŚNIAK, Markov's inequality and the existence of an extension operator for $C^{\infty}$ functions, J. Approx. Theory, 61 (1990), 106-117.

[16] T. RANSFORD, Potential theory in the complex plane, Cambridge University Press, 1995.

[17] E. B. SAFF AND V. TotiK, Logarithmic potentials with external fields, Springer-Verlag, 1997.

[18] M. TIDTEN, Kriterien für die Existenz, von Ausdehnungsoperatoren zu $\varepsilon(K)$ für kompakte Teilmenge $K$ von $\mathbb{R}$, Arch. Math., 40 (1983), 73-81. 
[19] V. TotiK, Markoff constants for Cantor sets, Acta Sci. Math. (Szeged), 60 (1995), 715-734. 